\title{
Numerical Investigation on Turbulent Flow and Heat Transfer of Helium-Xenon Gas Mixture in a Circular Tube
}

\author{
Biao Zhou $\left(\mathbb{D}\right.$, Han Zhang $\left(\mathbb{D}\right.$, Yu Ji $\mathbb{D}^{D}$, Jun Sun ${ }^{(D)}$, and Yuliang Sun ${ }^{(D)}$ \\ Institute of Nuclear and New Energy Technology, \\ Key Laboratory of Advanced Reactor Engineering and Safety of Ministry of Education, Tsinghua University, Beijing 100084, \\ China
}

Correspondence should be addressed to Biao Zhou; zhou-b17@mails.tsinghua.edu.cn and Jun Sun; sunjun@mail.tsinghua.edu.cn Received 23 April 2021; Accepted 18 August 2021; Published 31 August 2021

Academic Editor: Arkady Serikov

Copyright (c) 2021 Biao Zhou et al. This is an open access article distributed under the Creative Commons Attribution License, which permits unrestricted use, distribution, and reproduction in any medium, provided the original work is properly cited.

\begin{abstract}
Gas-cooled space nuclear reactor system usually utilizes the helium-xenon gas mixture as the working fluid. Since the typical helium-xenon mixture has the Prandtl number of about 0.2 , which is lower than that of water and air, the turbulent flow and heat transfer features need to be further investigated among the helium-xenon mixture and other fluids. In the current paper, numerical investigations by ANSYS Fluent are performed on helium-xenon mixture flow $(\mathrm{HeXe} 40, M=40.0 \mathrm{~g} / \mathrm{mol}, \mathrm{Pr}=0.21)$, airflow $(\operatorname{Pr}=0.71)$, and water flow $(\operatorname{Pr}=6.99)$ in the circular tube. Direct numerical simulation results of liquid metal flow $(\mathrm{Pr}=0.01)$ are also adopted for comparison. Results show that the dimensionless velocity profile and shear stress in the boundary layer of HeXe40 are close to those of other fluids. The empirical correlations from other fluids can also predict well the friction factor of helium-xenon mixtures. Due to the discrepancy in turbulent heat diffusivity ratio, the dimensionless radial temperature profile and turbulent heat conduction of HeXe40 significantly differ from those of other fluids. The molecular conduction region of HeXe40 develops up to $y^{+} \approx 30$ and extends to the logarithmic region of the flow boundary layer. Moreover, the available experimental Nusselt numbers of helium-xenon mixtures are compared with several convective heat transfer correlations, in which Kays correlation is better.
\end{abstract}

\section{Introduction}

Deep-space exploration and planetary outposts will become certain things in the near future [1]. These ambitious missions substantially require advanced and reliable space power. Compared with chemical batteries and solar photovoltaic arrays, the space nuclear reactor power is featured with a long working duration, wide power coverage, and being free from environmental impact, which is regarded as a promising technology for space exploration [2]. Among the existing roadmaps of technology, the gas-cooled nuclear reactor combined with a direct Brayton cycle is perceived as one of the most rational schemes for megawatt-class space nuclear reactor power systems [3]. For terrestrial gas-cooled nuclear reactor system, pure helium is widely adopted as the working fluid due to its chemical inertia and excellent thermophysical properties. However, its small molecular weight inevitably increases the stages of compressor and the aerodynamic loading of turbomachinery, which in turn increases the mass and volume of the system [4]. To reduce the size of the Brayton cycle unit, the helium-xenon gas mixtures with a molecular weight of $14.5-40.0 \mathrm{~g} / \mathrm{mol}$ are generally adopted as the working fluid [5].

As shown in Figure 1, the variation of Prandtl number for helium-xenon mixtures with different mixing ratios is presented. The value of the Prandtl number is found to be almost independent of temperature. Additionally, the notable feature of helium-xenon mixture in such concerned mixing-ratio range is the low Prandtl number, approximately $0.20-0.30$, which is obviously lower than that of conventional air and water $(\operatorname{Pr}>0.70)$ and is higher than that of liquid metal $(\operatorname{Pr} \cong 0.01)$. The Prandtl number determines the relative thickness of the flow boundary layer and thermal boundary layer and significantly affects the turbulence 


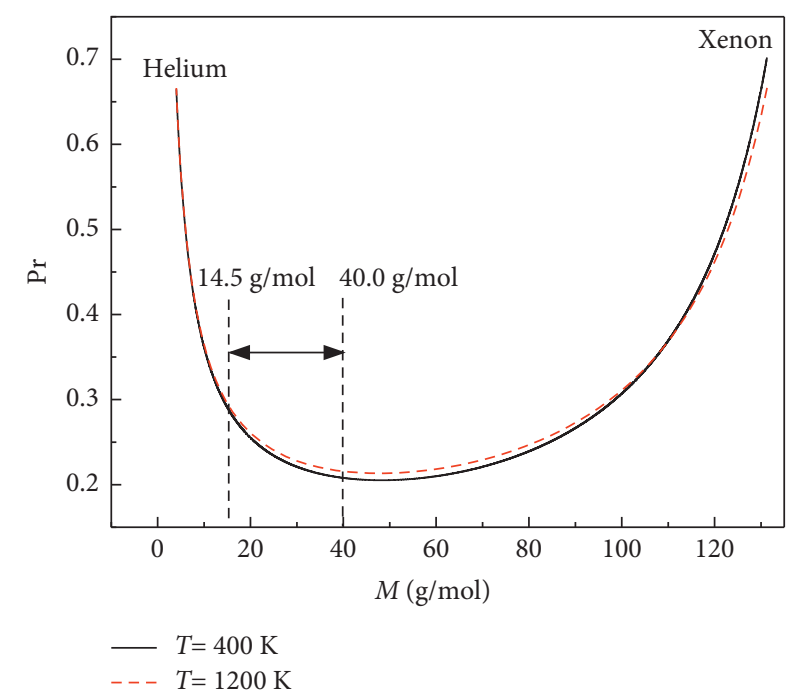

Figure 1: Variation of Prandtl number for helium-xenon gas mixtures at $P=2 \mathrm{MPa}$.

transport of momentum and heat [6]. For fluids with the Prandtl number closing to unity, the similarity between the velocity and temperature fields is valid. Based on the similarity, the classic Reynolds analogy method was proposed [7]. However, as the Prandtl number decreases, the flow boundary layer is gradually immersed in the thermal boundary layer, resulting in the failure of the similarity basis. Therefore, the Reynolds analogy is no longer applicable for fluids with a low Prandtl number [8]. To further investigate the effect of the Prandtl number on the turbulent convective heat transfer, many related experimental and numerical studies have been carried out. Gowen et al. [9] experimentally studied the effect of the Prandtl number on temperature profiles in a pipe and proposed a correlation of logarithmic temperature distribution. However, this study only focused on the fluids with a Prandtl number greater than unity. Kader et al. [10] conducted experiments of fluids with $0.026 \leq \operatorname{Pr} \leq 170$ in a heated tube and the turbulent flow and heat transfer was studied, but the gas mixtures with $0.1 \leq \operatorname{Pr} \leq 0.7$ are not involved. Besides, Taylor et al. [11] and Victosky et al. [12, 13] experimentally investigated the turbulent heat transfer of helium-xenon gas mixtures in pipes with different shapes, proving that the convective heat transfer of helium-xenon gas mixtures is different from that of conventional fluids. However, the mechanism of the differences has not been fully explained and detailed analysis has not been given either.

Since the velocity and temperature change drastically in the boundary layer, the experimental measurement in the limited space may inevitably bring large uncertainty. With the rapid development of computer technology, the numerical simulations have become an important tool for studying the turbulent flow and heat transfer. In the research of low-Pr fluids, Bricteux et al. [14] numerically studied the turbulent heat transfer of lead-bismuth alloys in simple channels through Large Eddy Simulation (LES) and Direct Numerical Simulation (DNS), visualizing the vortex structure of the low-Pr liquid metals. Duponcheel et al. [15] also conducted LES to investigate the convective heat transfer for liquid metals with different Prandtl numbers and evaluated the rationality of the current RANS model. Additionally, Shams et al. [16] carried out related numerical investigation on the turbulent heat transfer for the liquid lead and sodium. However, the above-mentioned researches mainly serve liquid metals, and other low-Pr fluids like gas mixtures were rarely involved. To study the turbulent transport characteristics of the fluids in a larger range of Prandtl numbers, Piller et al. [17] numerically investigated the turbulent heat transfer of fluids with five Prandtl numbers $(\mathrm{Pr}=1.0,0.3,0.1$, 0.05 , and 0.025$)$ in a simple channel under $\operatorname{Re}_{\tau}=150$ through DNS. The influence of Prandtl number on Reynolds stress transport and turbulent heat diffusion was discussed. Besides, Kawamura et al. [18] conducted DNS and investigated the turbulent heat transfer for fluids with $0.0025<\operatorname{Pr}<5.0$. However, limited by the huge amount of computation for DNS, the Reynolds number of the above cases was generally low. Since the law of turbulent heat transfer might be different as the change of Reynolds number, the universality of current conclusions from DNS to higher Reynolds number conditions remains to be studied. To better reveal the underlying mechanism of high-Re turbulent flow and heat transfer for low-Pr helium-xenon gas mixtures, it is necessary to carry out in-depth research.

By considering the wide use of $\mathrm{HeXe} 40$ in most schemes [19-22], numerical investigations on turbulent heat transfer of HeXe40 $(\mathrm{Pr}=0.21)$ in a heated tube are performed by employing the new modified turbulent Prandtl number $\left(\operatorname{Pr}_{t}\right)$ model proposed in our previous paper [23]. To better reflect the particularity of low-Pr helium-xenon mixture on the turbulent flow and heat transfer in the near-wall region, a numerical comparison between $\mathrm{HeXe} 40$ and air $(\mathrm{Pr}=0.71)$ and liquid water $(\mathrm{Pr}=6.99)$ is conducted. DNS results of velocity and temperature profiles for liquid metal $(\mathrm{Pr}=0.01)$ by Alcantara-Avila et al. [24, 25] are also introduced to compare. Thereafter, the differences between HeXe40 and other fluids are quantitatively described by analyzing the variation of turbulence parameters characterizing the turbulent momentum and heat transport in the boundary layer. Additionally, the mechanism of the differences in turbulent heat transfer is investigated at different transport scales. Finally, the applicability of the existing correlations for wall friction factor and Nusselt number to helium-xenon gas mixtures is also evaluated. This study can provide a certain reference for the subsequent thermal-hydraulic design of helium-xenon-cooled space nuclear reactors.

\section{Numerical Model and Its Validation}

2.1. Turbulence Model. The geometric calculation model in the present study refers to the test section of Taylor's experiment, through which the flow and heat transfer of a variety of binary gas mixtures including helium-xenon mixtures were studied. As shown in Figure 2, the test section is a straight tube and its inner diameter $(D)$ is $5.87 \mathrm{~mm}$. The first part $\left(l_{1}\right)$ of the tube is adiabatic and the remaining $\left(l_{2}\right)$ is heated by an electric resistance wire to obtain a uniform heat flux. 


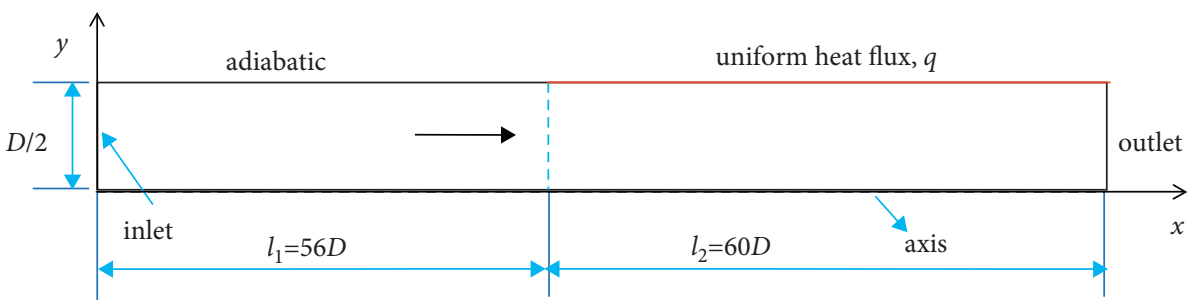

Figure 2: Test section of experiment for helium-xenon mixtures by Taylor et al.

Our previous article has investigated the applicability of different turbulence models to the numerical simulation of turbulent flow and heat transfer for helium-xenon gas mixtures. It is found that the SST $k-\omega$ model has a better prediction accuracy than other turbulence models. Therefore, the SST $k-\omega$ model is selected for numerical simulations in this study. Additionally, to ensure the validity of numerical calculation, the grid independence verification also has been studied in the literature [23]. The current mesh uses the grid number of $40 \times 2000$ with the $y^{+}$of the first grid less than 0.5 .

For RANS modeling, the main issue is the determination of the turbulent momentum diffusivity $\left(\varepsilon_{m}\right)$ and turbulent heat diffusivity $\left(\varepsilon_{h}\right)$. To make the governing equations closed, many models such as the mixing-length model have been proposed to calculate $\varepsilon_{m}$ based on the eddy viscosity assumption [26]. However, the value of $\varepsilon_{h}$ is difficult to predict directly by turbulence models. To solve this problem, the concept of turbulent Prandtl number is introduced. It may be helpful to understand the significance of $\operatorname{Pr}_{t}$ in the turbulent heat transfer simulations from the transport equations [27]. The governing equations with variable properties in a two-dimension cylindrical coordinate can be written as follows:

$$
\begin{aligned}
\frac{1}{r}\left[\frac{\partial}{\partial x}(r \rho \bar{u})+\frac{\partial}{\partial r}(r \rho \bar{v})\right] & =0, \\
\frac{1}{r}\left[\frac{\partial}{\partial x}(r \rho \overline{u u})+\frac{\partial}{\partial r}(r \rho \overline{u v})\right] & =-\frac{\partial P}{\partial x}+\frac{2}{r} \frac{\partial}{\partial x}\left(r \rho\left(\nu+\varepsilon_{m}\right) \frac{\partial \bar{u}}{\partial x}\right)+\frac{1}{r} \frac{\partial}{\partial r}\left[r \rho\left(\nu+\varepsilon_{m}\right)\left(\frac{\partial \bar{u}}{\partial r}+\frac{\partial \bar{v}}{\partial x}\right)\right]+\rho g, \\
\frac{1}{r}\left[\frac{\partial}{\partial x}(r \rho \overline{u v})+\frac{\partial}{\partial r}(r \rho \overline{v v})\right] & =-\frac{\partial P}{\partial r}+\frac{1}{r} \frac{\partial}{\partial x}\left[r \rho\left(\nu+\varepsilon_{m}\right)\left(\frac{\partial \bar{u}}{\partial r}+\frac{\partial \bar{v}}{\partial x}\right)\right]+\frac{2}{r} \frac{\partial}{\partial r}\left(r \rho\left(\nu+\varepsilon_{m}\right) \frac{\partial \bar{v}}{\partial r}\right)-\frac{2 \rho\left(\nu+\varepsilon_{m}\right) \bar{v}}{r^{2}}, \\
\frac{1}{r}\left[\frac{\partial}{\partial x}\left(r C_{p} \rho \overline{u T}\right)+\frac{\partial}{\partial r}\left(r C_{p} \rho \overline{v T}\right)\right] & =\frac{1}{r} \frac{\partial}{\partial x}\left[r C_{p} \rho\left(\alpha+\varepsilon_{h}\right) \frac{\partial \bar{T}}{\partial x}\right]+\frac{1}{r} \frac{\partial}{\partial r}\left[r C_{p} \rho\left(\alpha+\varepsilon_{h}\right) \frac{\partial \bar{T}}{\partial r}\right] .
\end{aligned}
$$

The expression of turbulent Prandtl number is described as

$$
\operatorname{Pr}_{t}=\frac{\varepsilon_{m}}{\varepsilon_{h}}
$$

Substituting equation (5) into (4), the energy equation becomes

$$
\frac{1}{r}\left[\frac{\partial}{\partial x}\left(r C_{p} \rho \overline{u T}\right)+\frac{\partial}{\partial r}\left(r C_{p} \rho \overline{v T}\right)\right]=\frac{1}{r} \frac{\partial}{\partial x}\left[r C_{p} \rho\left(\alpha+\frac{\varepsilon_{m}}{\operatorname{Pr}_{t}}\right) \frac{\partial \bar{T}}{\partial x}\right]+\frac{1}{r} \frac{\partial}{\partial r}\left[r C_{p} \rho\left(\alpha+\frac{\varepsilon_{m}}{\operatorname{Pr}_{t}}\right) \frac{\partial \bar{T}}{\partial r}\right]
$$

As shown in equation (6), we can easily identify the terms that influence heat transfer. The first term is $\left(C_{p} \rho \alpha\right)$, which represents the contribution of molecular conduction, whereas the second term, $\left(C_{p} \rho \varepsilon_{m} / \operatorname{Pr}_{t}\right)$, represents the turbulent-conduction contribution. Since the $\varepsilon_{m}$ can be just well predicted by a turbulence model, the following critical task of numerical simulations is the determination of a suitable $\operatorname{Pr}_{t}$ model.
A number of reviews and texts have been devoted to the $\operatorname{Pr}_{t}$ of different fluids [28-30]. As shown in Figure 3, the $\operatorname{Pr}_{t}$ is found to be close to a constant (0.85) for conventional fluids such as air or water. However, DNS simulations showed that the $\operatorname{Pr}_{t}$ is higher than unity at low Prandtl numbers and significantly related to the Prandtl number and $y^{+}$. The $\operatorname{Pr}_{t}$ of liquid metal with $\operatorname{Pr}=0.025$ is much larger than that of other 


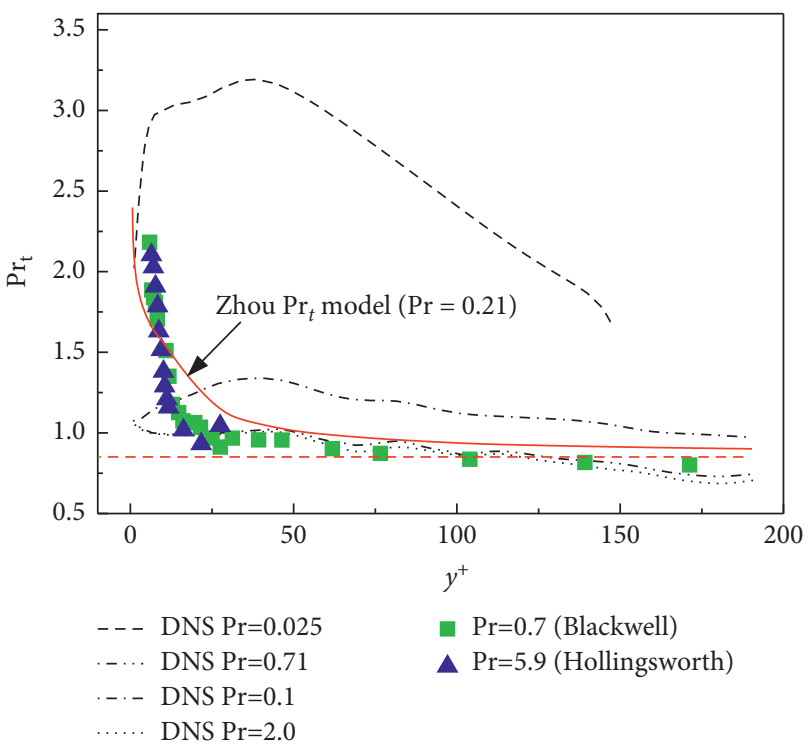

Figure 3: Variations of $\operatorname{Pr}_{t}$ for fluids with different Prandtl numbers.

fluids, which is mainly due to its smaller turbulent heat diffusivity. The Prandtl number of helium-xenon mixtures is between that of conventional fluids and liquid metals; thus, the existing $\operatorname{Pr}_{t}$ models might not be applicable to heliumxenon mixtures. To meet the requirement on numerical simulations of turbulent heat transfer for low-Pr heliumxenon mixtures, a modified three-parameter $\mathrm{Pr}_{t}$ model has been proposed in our previous article. In the present study, this $\mathrm{Pr}_{t}$ model will be implemented into ANSYS Fluent by user-defined functions (UDF) in numerical simulation of HeXe40. Additionally, $\operatorname{Pr}_{t}=0.85$ is adopted as the turbulent Prandtl number in simulations of air and water.

2.2. Thermophysical and Transport Properties. For gas-cooled space nuclear reactor system, the typical operating pressure is approximately $2 \mathrm{MPa}$ and the temperature range is roughly $400-1300 \mathrm{~K}$. Under these conditions, the theory of dilute gases based on the Chapman-Enskog approach is inapplicable. Tournier et al. [31] applied the law of corresponding states and proposed the semiempirical propertycalculation correlations for dense binary gas mixtures. Those correlations were verified by the experimental data across the pressure range of $0.1-20 \mathrm{MPa}$ and temperature up to $1400 \mathrm{~K}$. Based on the study of Tournier et al., a propertycalculation code for helium-xenon mixtures is developed. The calculations are shown in Figures 4-6. It indicates that the thermal conductivity $(\lambda)$ and dynamic viscosity $(\mu)$ increase with temperature, while their variation with pressure is almost negligible. The density $(\rho)$ is proportional to pressure but decreases as the temperature increases. Moreover, it is found that the specific heat capacity $\left(C_{p}\right)$ varies very slightly with temperature and pressure; thus, it can be regarded as a constant for a particular mixing ratio in numerical simulations. The property correlations except the specific heat capacity of helium-xenon mixture are implemented into ANSYS Fluent by UDF. The properties of air

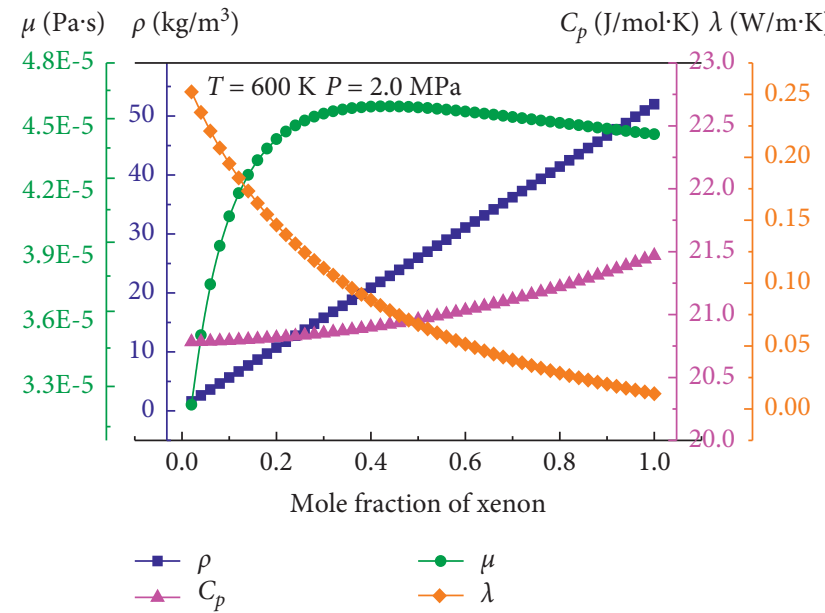

FIGURE 4: Variation of properties for helium-xenon mixtures with mole fraction of xenon.

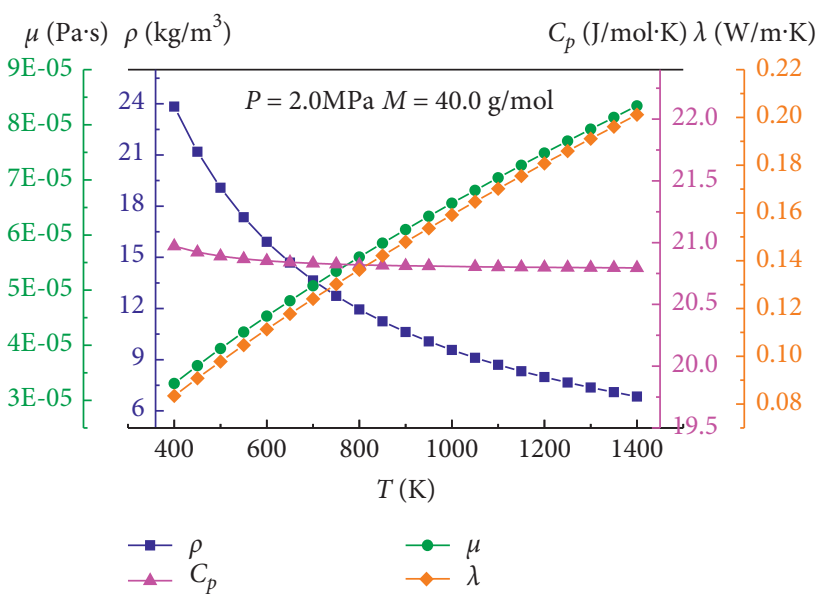

FIGURE 5: Variation of properties for helium-xenon mixture with temperature.

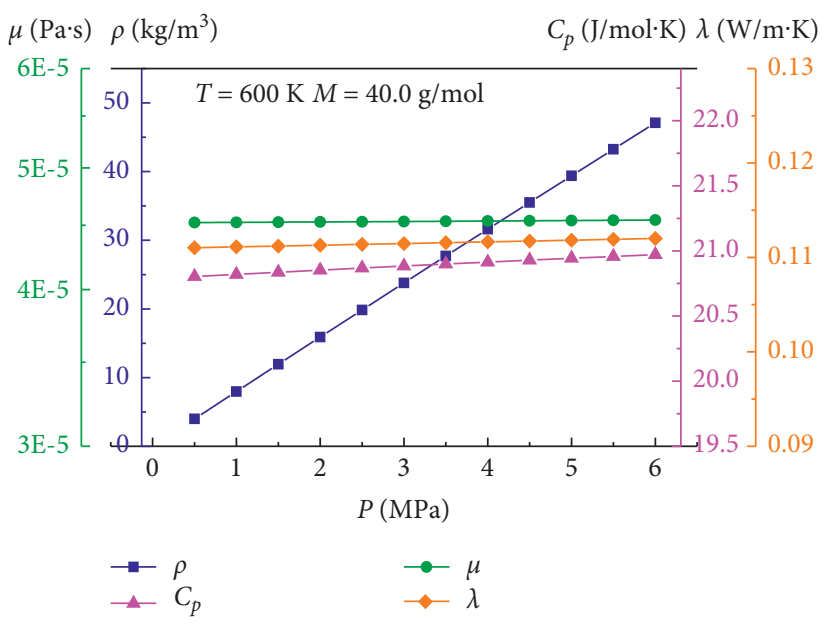

FIGURE 6: Variation of properties for helium-xenon mixture with pressure. 
and water refer to the default database materials in ANSYS Fluent.

2.3. Validation of Simulation Models. The applicability of the above model to helium-xenon mixtures has been verified in detail in the literature [23]. Hence, this section mainly focuses on the validation of simulation models for water and air. The mesh and solution methods of water and air simulation are the same as those of HeXe40 simulation. The simulated conditions for air and water are consistent with the relevant verification cases, respectively. Firstly, the DNS results of air under $\operatorname{Re}=48500$ are used to examine the simulation models. As shown in Figure 7, the profiles of velocity and temperature for air under the same Reynolds number in the fully developed region $(x=600 \mathrm{~mm})$ are calculated. Comparing the DNS results with numerical predictions, it can be found that the calculations of velocity and temperature for air are in good agreement with the results of DNS, thus verifying the rationality of the turbulence model in air simulation. Thereafter, the experimental data of Wei et al. [32] and Hollingsworth et al. are introduced to evaluate the turbulent model in the simulation of liquid water. As shown in Figure 8(a), the calculated velocity profile agrees well with the experimental data of Wei et al. under $\operatorname{Re}=39600$. Additionally, the temperature profile calculated in Figure 8(b) is also found to be basically consistent with the corresponding experimental data. Therefore, the applicability of the simulation model for liquid water is also verified. Next, the turbulent flow and heat transfer between low-Pr HeXe40 and other fluids will be quantitatively compared by analyzing the variation of turbulence parameters characterizing the turbulent momentum and heat transport by using the above models.

\section{Results and Discussion}

3.1. Characteristics of Turbulent Flow. The cross section of $x=600 \mathrm{~mm}$ is taken as the research object to investigate the characteristics of the turbulent flow for different fluids in the fully developed region. In terms of boundary conditions, the inlet is set to "mass-flow-inlet" with the $T_{\text {in }}=300 \mathrm{~K}$, the outlet is set to "pressure-out" with $P_{\text {out }}=0.93 \mathrm{MPa}$, and the wall surface adopts a nonslip boundary with heat flux $q_{w}=7366 \mathrm{~W} / \mathrm{m}^{2}$. The mass flow rate of the inlet is adjusted for different fluids to ensure the same Reynolds number at the section of $x=600 \mathrm{~mm}$. As shown in Figure 9, the velocity profiles of fluids under $\mathrm{Re}=39600$ are calculated. The DNS results of liquid metal, of which the bulk Reynolds number is also close to $\mathrm{Re}=39600$, are introduced for comparison. Figure 9 shows that the velocity profile of HeXe40 almost coincides with those of other fluids. Moreover, there exists an obvious logarithmic law of velocity, which agrees well with the predictions of the common Nikuradse equation. The above results indicate that the velocity profiles of fluids are almost independent of the Prandtl number.

The momentum transport of fluid is achieved through viscous force. According to different scales, it can be divided into molecular shear stress caused by molecular motion and turbulent shear stress caused by the motion of fluid micelles. Comparing the variation of momentum transport is helpful to reveal the characteristics of the turbulent flow for heliumxenon mixture and the fluids with other Prandtl numbers. Therefore, the dimensionless molecular shear stress $\left(\bar{\tau}_{m}^{+}\right)$, turbulent shear stress $\left(\bar{\tau}_{t}^{+}\right)$, and total shear stress $\left(\bar{\tau}^{+}\right)$of various fluids are calculated in the present study. As shown in Figure 10, the variation of $\bar{\tau}_{m}^{+}$and $\bar{\tau}_{t}^{+}$are found to be close to each other, indicating that the momentum transport of HeXe40 is similar to that of other fluids. Additionally, the intersection points of $\bar{\tau}_{m}^{+}$and $\bar{\tau}_{t}^{+}$for different fluids are coincident around $y^{+}=11$; that is, the molecular momentum transport is equivalent to the turbulent momentum transport at this position. Therefore, $y^{+}=11 \mathrm{can}$ be regarded as the demarcation point between the linear region and logarithmic region in the flow boundary layer. Moreover, Kays et al. [7] and Schlichting et al. [33] experimentally found that the demarcation points of flow boundary layer for water and air were also approximately $y^{+}=11$. Therefore, it is illustrated that the turbulent flow characteristics of HeXe40 are not obviously different from conventional fluids and liquid metals.

Obtaining an appropriate wall friction factor $(f)$ is one of the main issues of studying the characteristics of fluid flow. For conventional fluids such as water and air, many empirical friction factor correlations have been proposed. As shown in Table 1, four common correlations [34-36] are selected to calculate the wall friction factor of helium-xenon mixtures. The calculations by various correlations are compared with the results of CFD simulation and experimental data [11]. As shown in Figure 11, the simulated results are very close to the calculations of four empirical correlations and agree well with the experimental data, which further reflects the similarity in the turbulent flow characteristics of the helium-xenon mixtures and the conventional fluids.

3.2. Characteristics of Turbulent Heat Transfer. As shown in Figure 12, the radial temperature profiles of $\mathrm{HeXe} 40$ and other fluids are calculated. It is found that the temperature profiles of various fluids are significantly different. As the Prandtl number decreases, the temperature profile becomes smoother, and the similarity between velocity profile and temperature profile gradually disappears. When $\operatorname{Pr}=0.01$, there is no obvious temperature logarithmic law. The temperature profile of $\mathrm{HeXe} 40$ is between that of air and liquid metal, and the overall trend of HeXe40 is closer to that of air. Different from the liquid metal, a temperature logarithmic law can be found for low-Pr HeXe40.

The heat transport of fluids is realized by heat conduction. Similar to the momentum transport, the heat transport can be divided into molecular heat conduction and turbulent heat conduction, which are caused by the molecular thermal motion and the pulsation of fluid micelles, respectively. To compare the characteristics of turbulent heat transfer between the helium-xenon mixture and other fluids, the dimensionless molecular heat conduction $\left(\bar{q}_{m}^{+}\right)$, turbulent heat conduction $\left(\bar{q}_{t}^{+}\right)$, and total heat flux $\left(\bar{q}^{+}\right)$of various 


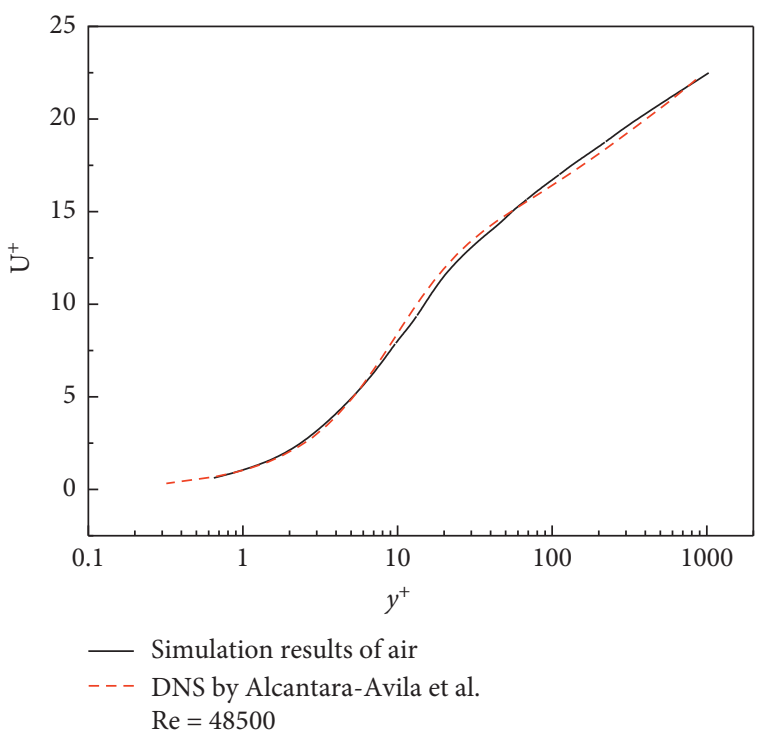

(a)

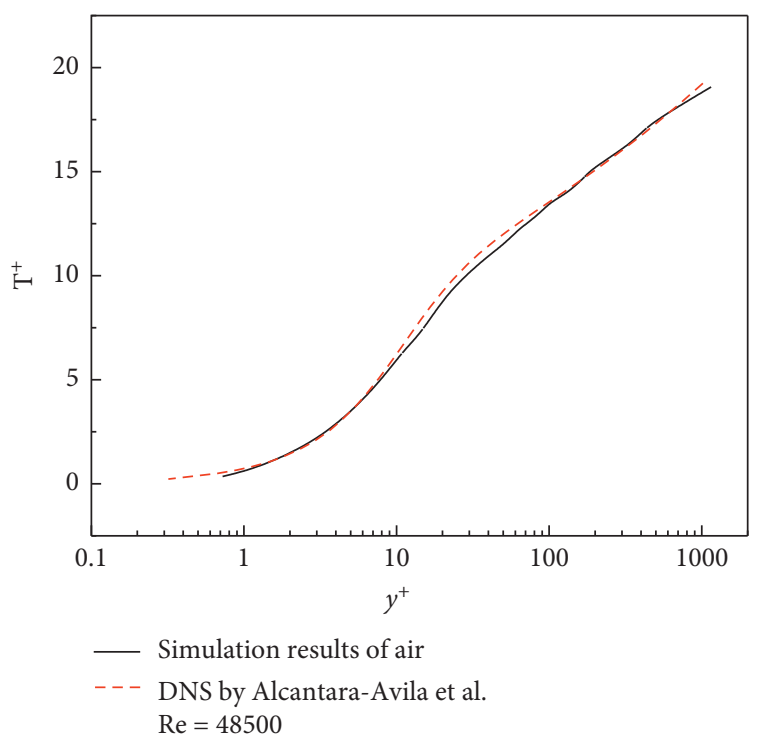

(b)

FIgURe 7: Comparison of air numerical simulations and DNS results. (a) Profile of air velocity and (b) profile of air temperature.

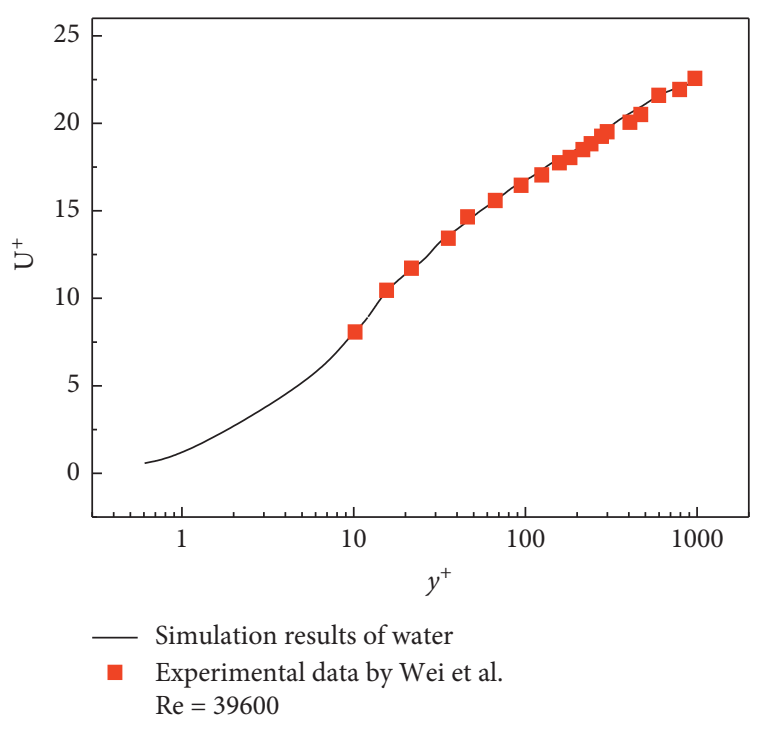

(a)

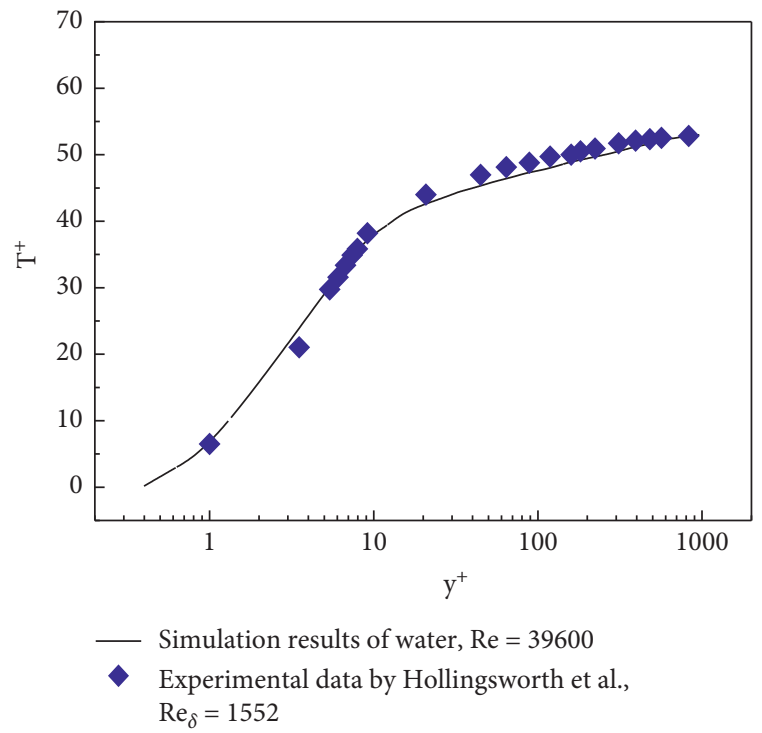

(b)

Figure 8: Comparison of water numerical simulations and experimental data. (a) Profile of water velocity and (b) profile of water temperature.

fluids are calculated. As shown in Figure 13, in terms of molecular heat conduction, the smaller the Prandtl number is, the larger $\bar{q}_{m}^{+}$is, indicating that molecular heat conduction is gradually prominent as the Prandtl number decreases. The $\bar{q}_{m}^{+}$profile of HeXe40 is significantly lower than that of liquid metal and is closer to that of the air. However, it is found that the value of $\bar{q}_{m}^{+}$of HeXe40 is still more than twice that of air in the region of $15<y^{+}<200$.

In terms of the turbulent heat conduction, $\bar{q}_{t}^{+}$first increases and then decreases as $y^{+}$increases, which is owing to the fact that the variation of radial temperature gradient and turbulent thermal diffusivity with $y^{+}$is always opposite. Moreover, as the Prandtl number decreases, the value of $\bar{q}_{t}^{+}$decreases, and the farther the peak of $\bar{q}_{t}^{+}$is from the wall. Under the working condition studied in the present paper, the maxima of $\bar{q}_{t}^{+}$of the HeXe40 is 0.83 times that of air and 3.9 times that of the liquid metal. Different from the flow boundary layer, the intersection points of $\bar{q}_{m}^{+}$and $\bar{q}_{t}^{+}$of different fluids are found to be not fixed and vary with the Prandtl number. As shown in Figure 13, the demarcation 


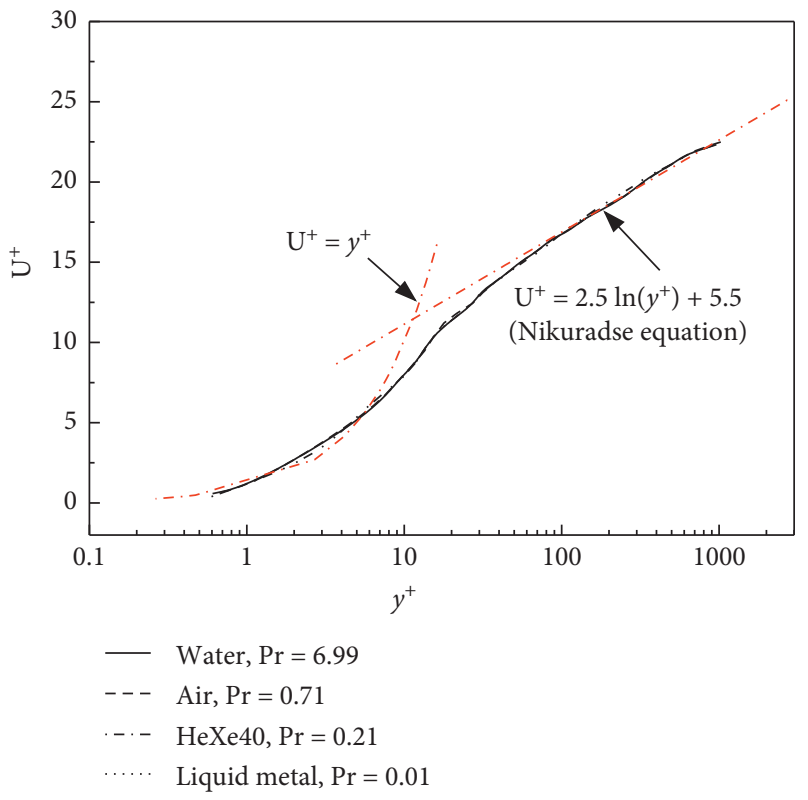

Figure 9: Velocity profiles of fluids with different Prandtl numbers.

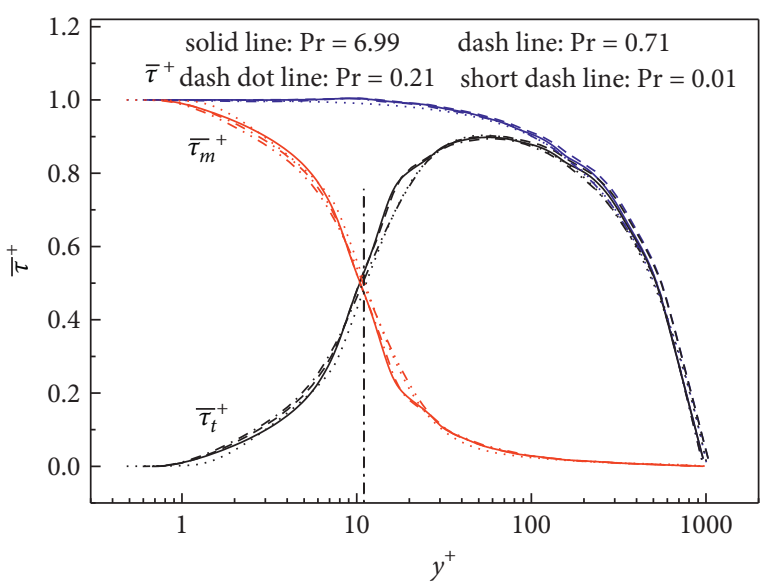

FIGURE 10: Dimensionless molecular shear stress $\left(\bar{\tau}_{m}^{+}=\mathrm{d} u^{+} / \mathrm{d} y^{+}\right)$, turbulent shear stress $\left(\bar{\tau}_{t}^{+}={\overline{u^{\prime}}}^{\prime}{ }^{+}\right)$, and total shear stress $\left(\bar{\tau}^{+}=\bar{\tau}_{m}^{+}+\bar{\tau}_{t}^{+}\right)$of different fluids.

TABle 1: Common correlations of wall friction factor.

\begin{tabular}{lcr}
\hline Name & Correlations & Range of application \\
\hline Blasius & $f=0.316 / \operatorname{Re}^{0.25}$ & $5.0 \cdot 10^{3} \leq \operatorname{Re} \leq 10^{5}$ \\
Colebrook & $1 / \sqrt{f}=1.81 \mathrm{R}(\operatorname{Re} / 6.9)$ & $5.0 \cdot 10^{3} \leq \operatorname{Re} \leq 5.0 \cdot 10^{7}$ \\
Drewr & $f=0.0056+0.5 \cdot \operatorname{Re}^{-0.32}$ & $3.0 \cdot 10^{3} \leq \operatorname{Re} \leq 3.0 \cdot 10^{6}$ \\
Taitel & $f=0.184 / \operatorname{Re}^{0.2}$ & $\operatorname{Re} \geq 3.0 \cdot 10^{3}$ \\
\hline
\end{tabular}

point of air is $y^{+}=11.4$, indicating that the molecular conduction region of the thermal boundary layer nearly coincides with the linear region of the flow boundary layer. However, the demarcation point of HeXe40 is $y^{+}=29.6$, which indicates that the molecular conduction region has extended to the logarithmic region of flow boundary layer. For the liquid metal, even at the center of the flow channel, $\bar{q}_{t}^{+}$is still smaller than $\bar{q}_{m}^{+}$, indicating that its turbulent heat transport is almost negligible.

To further analyze the discrepancy in the turbulent heat transport of different fluids, the variation of the turbulent heat diffusivity ratio $\left(\varepsilon_{h} / \alpha\right)$ is calculated. As shown in Figure 14 , the value of $\varepsilon_{h} / \alpha$ is positively associated with the Prandtl number. That indicates that as the Prandtl number 


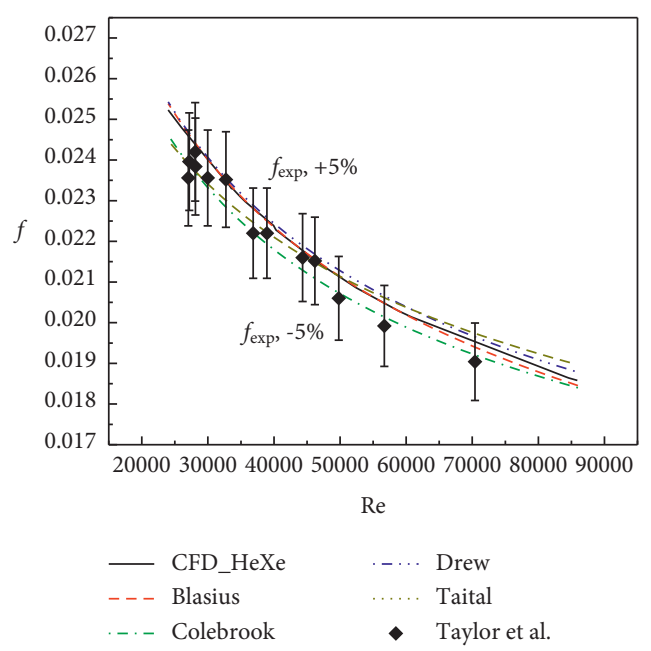

FIGURE 11: Comparison of calculated wall friction factor with experimental data of helium-xenon mixtures.

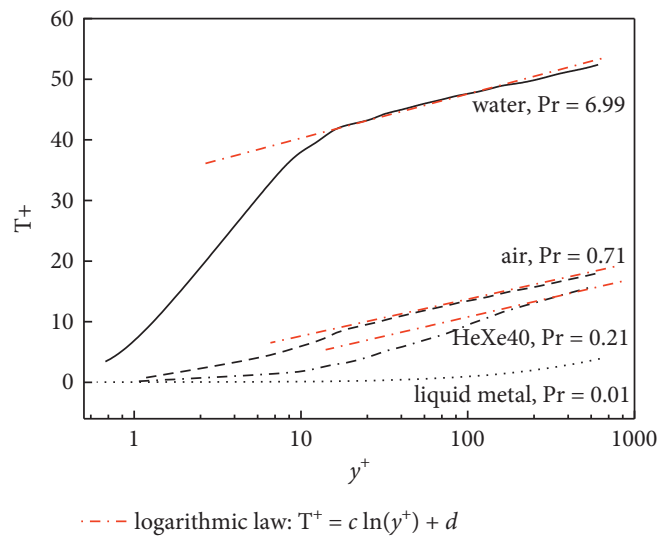

FIGURE 12: Temperature profiles of various fluids.

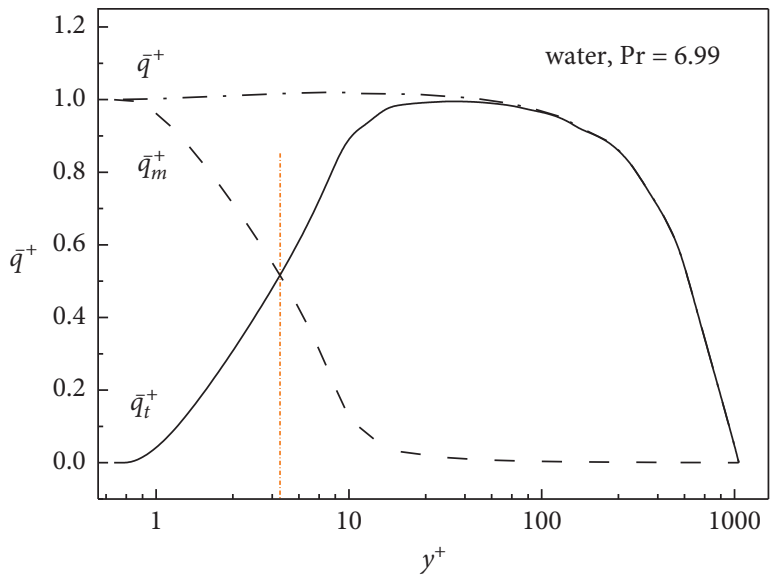

(a)

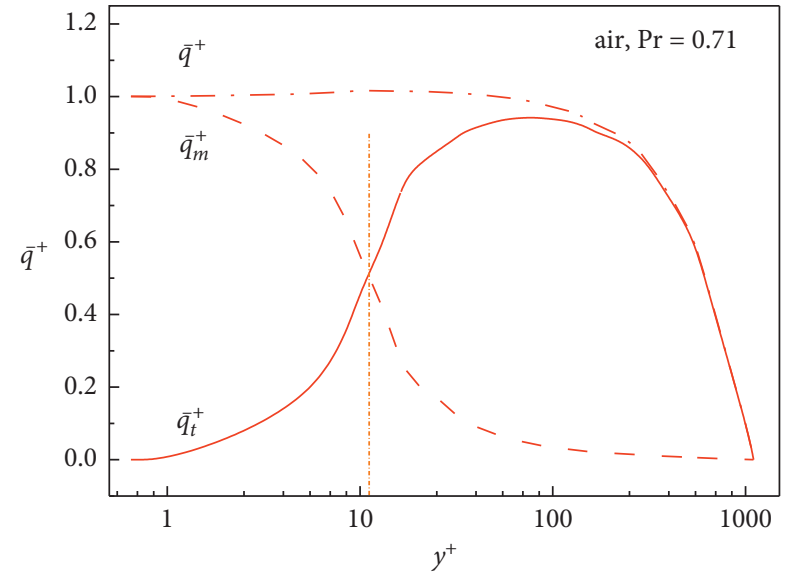

(b)

Figure 13: Continued. 


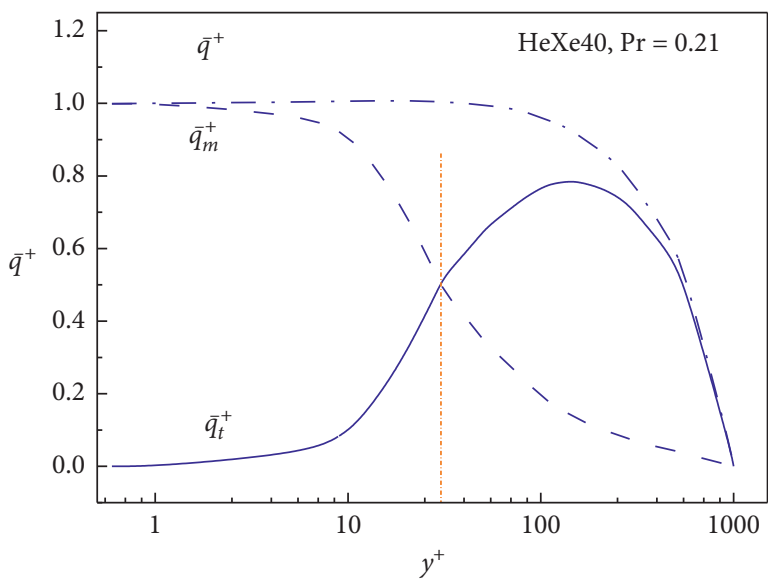

(c)

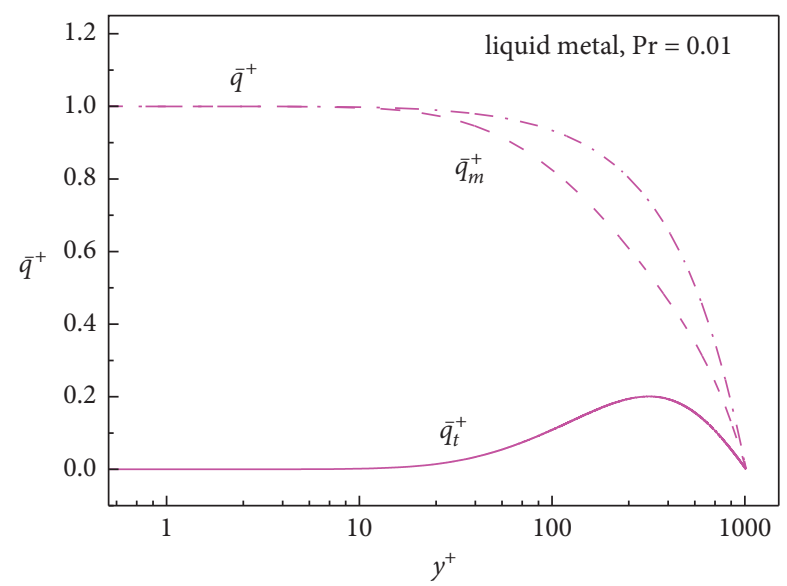

(d)

Figure 13: Dimensionless molecular heat conduction $\left(\bar{q}_{m}^{+}=\operatorname{Pr}^{-1} \mathrm{~d} T^{+} / \mathrm{d} y^{+}\right)$, turbulent heat conduction $\left(\bar{q}_{t}^{+}={\overline{v^{\prime} T^{\prime}}}^{+}\right)$, and total heat flux $\left(\bar{q}^{+}=\bar{q}_{m}^{+}+\bar{q}_{t}^{+}\right)$of different fluids.

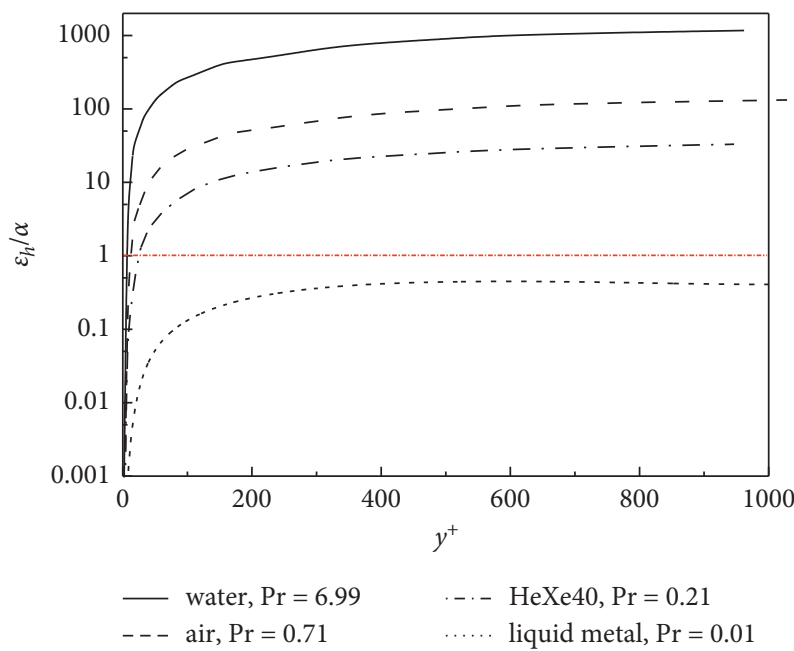

Figure 14: Variation in turbulent heat diffusivity ratio of different fluids.

decreases, the thermal diffusion by turbulent pulsation of fluid micelles is weakened and is gradually transported by molecular thermal motion between different temperature layers. Although the $\varepsilon_{h} / \alpha$ of HeXe40 is significantly smaller than that of air and water, it is greater than 1.0 starting from the near-wall region of $y^{+}>30$, indicating that the heat transfer of HeXe40 is still dominated by turbulent heat conduction. Based on the above analyses, it can be seen that the mechanism of turbulent heat transfer of HeXe40 is different from that of conventional air and water and extremely low-Pr liquid metals.

Nusselt number is an important dimensionless parameter to characterize the convective heat transfer. Based on the experimental data of water, air, and liquid metal, and so on, a large number of empirical Nusselt number correlations have been proposed. To quantify the difference in turbulent heat transfer of various fluids, several common Nusselt number correlations are selected in Table 2 [37-39] to evaluate their applicability to the studied helium-xenon mixtures $(0.2 \leq \operatorname{Pr} \leq 0.3)$.

As shown in Figure 15, the experimental data of binary gas mixtures including helium-xenon mixtures by Taylor et al. are introduced for comparison. Results show that the Dittus-Boelter (DB) correlation and Colburn correlation, which were obtained by experimental data of medium and high Prandtl number fluids, obviously overpredict the Nusselt number of helium-xenon mixtures. However, the correlations of Churchill, Stromquist, and Lyon, which are suitable for liquid metals, are found to underestimate the experimental data. The Kays correlation is proposed for gases in the range of $0.5 \leq \operatorname{Pr} \leq 2.0$. The calculations by Kays correlation are relatively better in agreement with the experimental Nusselt number. However, when $\mathrm{Re}=84000$, the predictions by Kays correlation are still slightly higher than 
TABLE 2: Existing common Nusselt number correlations.

\begin{tabular}{|c|c|c|}
\hline Name & Correlations & Scope of application \\
\hline Dittus-Boelter & $\mathrm{Nu}=0.021 \mathrm{Re}^{0.8} \operatorname{Pr}^{0.4}$ & $0.7 \leq \operatorname{Pr} \leq 10$ \\
\hline Colburn & $\begin{array}{l}\mathrm{Nu}=0.023 \operatorname{Re}^{0.8} \operatorname{Pr}^{1 / 3} \\
\mathrm{Nu}=5+0.015 \operatorname{Re}^{m} \operatorname{Pr}^{n}\end{array}$ & $0.5 \leq \operatorname{Pr} \leq 100$ \\
\hline Sleicher & $\begin{array}{l}m=0.88-0.24 /(4+\operatorname{Pr}) \\
n=1 / 3+0.5 \exp (0.6 \operatorname{Pr})\end{array}$ & $0.1 \leq \operatorname{Pr} \leq 10^{5}$ \\
\hline Kays & $\mathrm{Nu}=0.022 \operatorname{Re}^{0.8} \operatorname{Pr}^{0.6}$ & $0.5 \leq \operatorname{Pr} \leq 1.0$ \\
\hline Churchill & $\begin{array}{l}\mathrm{Nu}=0.4977 \operatorname{Re} \sqrt{f} \operatorname{Pr} /\left(1+\operatorname{Pr}^{0.8}\right)^{5 / 6} \\
1 / \sqrt{f}=2.21 \cdot \ln (\operatorname{Re} / 7)\end{array}$ & $0.001 \leq \operatorname{Pr} \leq 200$ \\
\hline Stromquist & $\mathrm{Nu}=3.6+0.025 \mathrm{Pe}^{0.8}$ & $88 \leq \mathrm{Pe} \leq 4000$ \\
\hline Lyon & $\mathrm{Nu}=7.0+0.025(\mathrm{Pe} / 2)^{0.8}$ & $\operatorname{Pr}<0.1$ \\
\hline
\end{tabular}

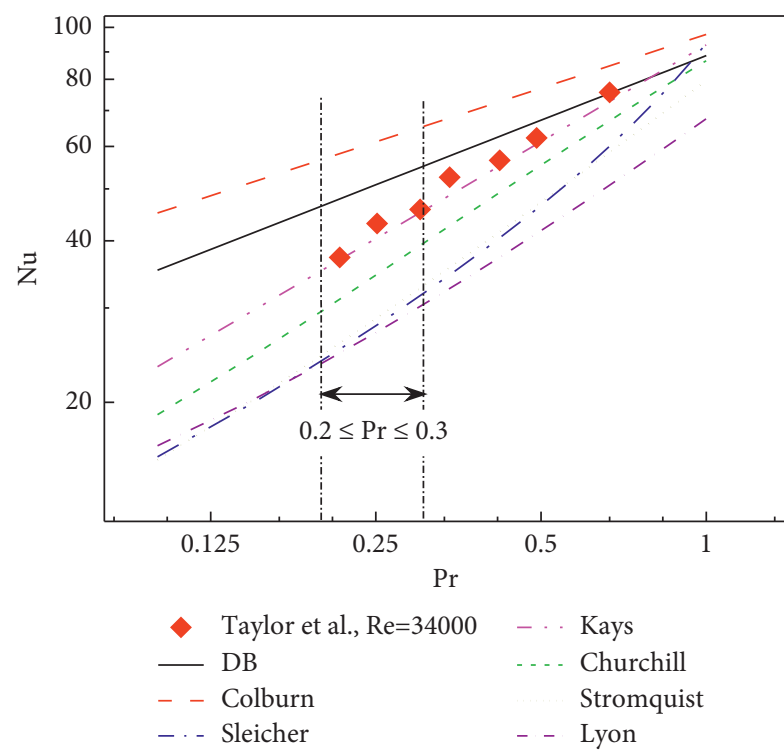

(a)

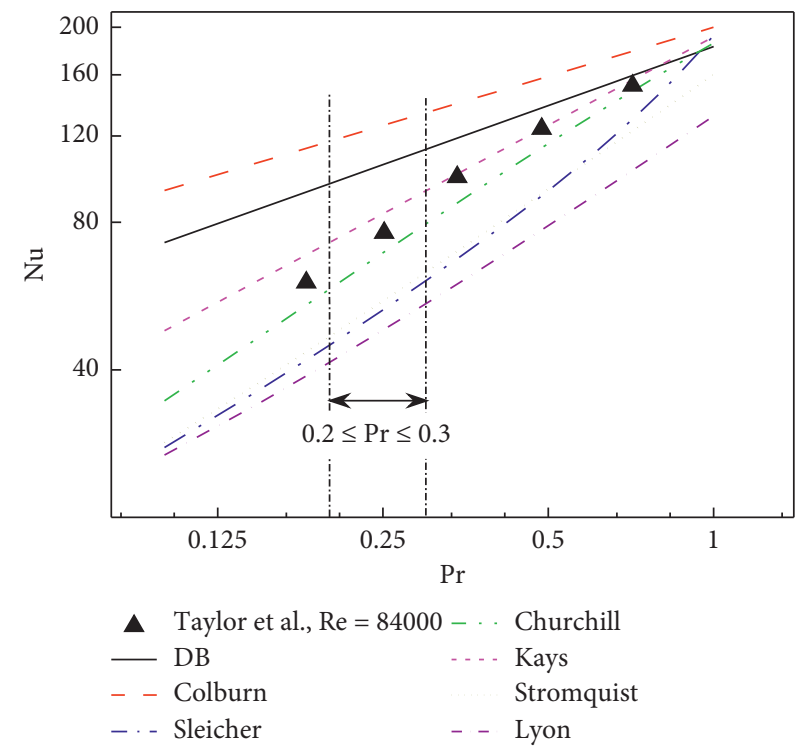

(b)

Figure 15: Comparison of calculations by Nusselt number correlations and experimental data.

the experimental data of $0.2 \leq \operatorname{Pr} \leq 0.3$. The above results are mainly due to the fact that the fluid with a larger Prandtl number has a larger turbulent heat diffusivity at the same Reynolds number, thus bringing about a higher heat transfer intensity and a larger Nusselt number.

\section{Conclusions}

Numerical investigations are conducted by using the new modified $\mathrm{Pr}_{t}$ model to study the turbulent flow and heat transfer of helium-xenon mixture in a circular tube. By comparing the distributions of the dimensionless velocity and temperature in the boundary layer, the differences in turbulent heat transfer between the HeXe40 and other fluids are described. Additionally, considering the formation mechanism of turbulent momentum transport and heat transport, the reason explaining the differences is revealed by analyzing the variation of dimensionless shear stress and heat flux under two different transport scales. What is more, the applicability of existing correlations on the wall friction factor and Nusselt number are evaluated for low-Pr heliumxenon mixtures. The following conclusions can be drawn:

(1) The velocity profiles and the shear stress variation of different fluids are similar, and the empirical correlations of friction factor used for conventional fluids are found to have good predictions on heliumxenon mixtures, indicating that the turbulent flow characteristics for helium-xenon mixtures are consistent with those of other fluids.

(2) Due to the discrepancy in turbulent heat diffusivity ratio, the mechanism of convective heat transfer of HeXe40 is different from that of air, water, and liquid metal. Although the molecular conduction region of $\mathrm{HeXe} 40$ extends to the logarithmic region of the flow boundary layer, the turbulent heat conduction is still dominant.

(3) Among all the selected Nusselt number correlations, the results by Kays correlation are found to be in better agreement with experimental data of heliumxenon gas mixtures. 
In the future, additional experimental studies are planned to investigate the flow and heat transfer of heliumxenon mixtures in pipes with various geometries. More applicable heat transfer correlations will also be proposed with the assistance of the theoretical derivation and numerical fitting of related experimental data.

\section{Data Availability}

The data used to support the findings of this study are included within the article.

\section{Conflicts of Interest}

The authors declare that there are no conflicts of interest regarding the publication of this paper.

\section{Acknowledgments}

This project was supported by the CNSA Program (D010501) and China Postdoctoral Science Foundation (Grant no. 2020M680586).

\section{References}

[1] B. Zhou, Y. Ji, J. Sun, and Y. L. Sun, "Research review on space nuclear reactor power demand analysis," Atomic Energy Science and Technology, vol. 54, no. 10, pp. 1912-1923, 2020, in Chinese.

[2] T. Meng, K. Cheng, C. Zeng, Y. He, and S. Tan, "Preliminary control strategies of megawatt-class gas-cooled space nuclear reactor with different control rod configurations," Progress in Nuclear Energy, vol. 113, pp. 135-144, 2019.

[3] M. S. El-Genk and J.-M. Tournier, "Noble gas binary mixtures for gas-cooled reactor power plants," Nuclear Engineering and Design, vol. 238, no. 6, pp. 1353-1372, 2008.

[4] M. S. El-Genk and J. P. Tournier, "Properties of helium, nitrogen and He- $\mathrm{N}_{2}$ binary gas mixtures," Journal of Thermophysics and Heat Transfer, vol. 22, no. 3, pp. 442-456, 2008.

[5] Y. G. Dragunov, E. L. Romadova, B. A. Gabaraev, M. S. Belyakov, and D. S. Derbenev, "Recommendations on calculation of transport coefficients and thermodynamic properties of helium-xenon gas mixtures," Nuclear Engineering and Design, vol. 354, Article ID 110196, 2019.

[6] R. Verzicco and R. Camussi, "Prandtl number effects in convective turbulence," Journal of Fluid Mechanics, vol. 383, pp. 55-73, 1999.

[7] W. M. Kays, M. E. Crawford, and B. Weigand, Convective Heat and Mass Transfer, McGraw-Hill, New York, NY, USA, 4th edition, 2005.

[8] O. Zier, W. Zimmermann, and W. Pesch, "On low-Prandtlnumber convection in an inclined layer of liquid mercury," Journal of Fluid Mechanics, vol. 874, pp. 76-101, 2019.

[9] R. A. Gowen and J. W. Smith, "The effect of the Prandtl number on temperature profiles for heat transfer in turbulent pipe flow," Chemical Engineering Science, vol. 22, no. 12, pp. 1701-1711, 1967.

[10] B. A. Kader, "Temperature and concentration profiles in fully turbulent boundary layers," International Journal of Heat and Mass Transfer, vol. 24, no. 9, pp. 1541-1544, 1981.

[11] M. F. Taylor, K. E. Bauer, and D. M. McEligot, "Internal forced convection to low-Prandtl-number gas mixtures,"
International Journal of Heat and Mass Transfer, vol. 31, no. 1, pp. 13-25, 1988.

[12] O. V. Vitovsky, M. S. Makarov, V. E. Nakoryakov, and V.S. Naumkin, "Heat transfer in a small diameter tube at high Reynolds numbers," International Journal of Heat and Mass Transfer, vol. 109, pp. 997-1003, 2017.

[13] O. V. Vitovsky, S. L. Elistratov, M. S. Makarov, V. E. Nakoryakov, and V. S. Naumkin, "Heat transfer in a flow of gas mixture with low Prandtl number in triangular channels," Journal of Engineering and Thermophysics, vol. 25, no. 1, pp. 15-23, 2016.

[14] L. Bricteux, M. Duponcheel, G. Winckelmans, I. Tiselj, and Y. Bartosiewicz, "Direct and large eddy simulation of turbulent heat transfer at very low Prandtl number: application to lead-bismuth flows," Nuclear Engineering and Design, vol. 246, pp. 91-97, 2012.

[15] M. Duponcheel, L. Bricteux, M. Manconi, G. Winckelmans, and Y. Bartosiewicz, "Assessment of RANS and improved near-wall modeling for forced convection at low Prandtl numbers based on LES up to $\operatorname{Re}_{\tau}=2000$," International Journal of Heat and Mass Transfer, vol. 75, pp. 470-482, 2014.

[16] A. Shams and A. De Santis, "Towards the accurate prediction of the turbulent flow and heat transfer in low-Prandtl fluids," International Journal of Heat and Mass Transfer, vol. 130, pp. 290-303, 2019.

[17] M. Piller, E. Nobile, and T. J. Hanratty, "DNS study of turbulent transport at low Prandtl numbers in a channel flow," Journal of Fluid Mechanics, vol. 458, pp. 419-441, 2002.

[18] H. Kawamura, K. Ohsaka, H. Abe, and K. Yamamoto, "DNS of turbulent heat transfer in channel flow with low to medium-high Prandtl number fluid," International Journal of Heat and Fluid Flow, vol. 19, no. 5, pp. 482-491, 1998.

[19] M. S. El-Genk, J.-M. P. Tournier, and B. M. Gallo, "Dynamic simulation of a space reactor system with closed Brayton cycle loops," Journal of Propulsion and Power, vol. 26, no. 3, pp. 394-406, 2010.

[20] J. C. King and M. S. El-Genk, "Thermal-hydraulic and neutronic analyses of the submersion-subcritical, safe space (S4) reactor," Nuclear Engineering and Design, vol. 239, no. 12, pp. 2809-2819, 2009.

[21] Z. Li, J. Sun, M. Liu, M. Lang, and L. Shi, "Design of a hundred-kilowatt level integrated gas-cooled space nuclear reactor for deep space application," Nuclear Engineering and Design, vol. 361, Article ID 110569, 2020.

[22] M. S. El-Genk and T. M. Schriener, "Long operation life reactor for lunar surface power," Nuclear Engineering and Design, vol. 241, no. 6SI, pp. 2339-2352, 2011.

[23] B. Zhou, Y. Ji, J. Sun, and Y. Sun, "Modified turbulent Prandtl number model for helium-xenon gas mixture with low Prandtl number," Nuclear Engineering and Design, vol. 366, Article ID 110738, 2020.

[24] F. Alcántara-Ávila, S. Hoyas, and M. J. Pérez-Quiles, “DNS of thermal channel flow up to $\mathrm{Re}_{\tau}=2000$ for medium to low Prandtl numbers," International Journal of Heat and Mass Transfer, vol. 127, pp. 349-361, 2018.

[25] F. Lluesma-Rodríguez, S. Hoyas, and M. J. Perez-Quiles, "Influence of the computational domain on DNS of turbulent heat transfer up to $\mathrm{Re}_{\tau}=2000$ for $\mathrm{Pr}=0.71$," International Journal of Heat and Mass Transfer, vol. 122, pp. 983-992, 2018.

[26] K. Bremhorst and L. Krebs, "Eddy diffusivity based comparisons of turbulent Prandtl number for boundary layer and free jet flows with reference to fluids of very low Prandtl number," Journal of Heat Transfer, vol. 115, no. 3, pp. 549-552, 1993. 
[27] G. Tang, H. Shi, Y. Wu et al., "A variable turbulent Prandtl number model for simulating supercritical pressure $\mathrm{CO} 2$ heat transfer," International Journal of Heat and Mass Transfer, vol. 102, pp. 1082-1092, 2016.

[28] D. K. Hollingsworth, W. M. Kays, and R. J. Moffat, "The measurement and prediction of heat transfer in a turbulent boundary layer in water," in Proceedings of the 7th Symposium on Turbulent Shear Flows, Stanford, CA, USA, August 1989.

[29] B. F. Blackwell, W. M. Kays, and R. J. Moffat, "The turbulent boundary layer on a porous plate an experimental study of the heat transfer behavior with adverse pressure gradients," Report no. NASA-CR-130291, Stanford University, Stanford, CA, USA, 1972.

[30] W. M. Kays, "Turbulent Prandtl number-where are we?," Journal of Heat Transfer, vol. 116, no. 2, pp. 284-295, 1994.

[31] J. P. Tournier, M. S. El-Genk, and B. M. Gallo, "Best estimates of binary gas mixtures properties for closed Brayton cycle space applications," in Proceedings of the 4th International Energy Conversion Engineering Conference and Exhibit (IECEC), San Diego, CA, USA, June 2006.

[32] T. Wei and W. W. Willmarth, "Reynolds-number effects on the structure of a turbulent channel flow," Journal of Fluid Mechanics, vol. 204, no. 1, pp. 57-95, 1989.

[33] H. Schlichting and K. Gersten, Boundary-Layer Theory, Springer, New York, NY, USA, 9th edition, 2017.

[34] Y. Taitel and A. E. Dukler, "A model for predicting flow regime transitions in horizontal and near horizontal gasliquid flow," AIChE Journal, vol. 22, no. 1, pp. 47-55, 1976.

[35] C. F. Colebrook and C. M. White, "Experiments with fluid friction in roughened pipes," Proceedings of the Royal Society of London, Series A, Mathematical and Physical Sciences, vol. 161, no. A906, pp. 367-381, 1937.

[36] H. Blasius, "Law of similarity in fluid friction phenomena," VDI Zeitschrift, vol. 56, pp. 639-643, 1912.

[37] F. Chen, X. Huai, J. Cai, X. Li, and R. Meng, "Investigation on the applicability of turbulent-Prandtl-number models for liquid lead-bismuth eutectic," Nuclear Engineering and Design, vol. 257, pp. 128-133, 2013.

[38] R. N. Lyon, "Liquid metal heat transfer coefficients," Chemical Engineering Progress, vol. 47, no. 2, pp. 75-79, 1951.

[39] Y. G. Dragunov, V. P. Smetannikov, B. A. Gabaraev, M. S. Belyakov, and P. V. Kobzev, "On the choice of correlations for calculating the heat transfer coefficient in binary gas mixtures," Journal of Engineering and Thermophysics, vol. 22, no. 1, pp. 30-42, 2013. 